\title{
A NOTE ON WIMAN AND ACCOLA-MACLACHLAN SURFACES
}

\author{
Ravi S. Kulkarni
}

\section{Introduction}

A wellknown result of Wiman concerning automorphisms of Riemann surfaces is the fact that for $g \geq 2$ the largest cyclic group of automorphisms of a compact Riemann surface of genus $g$ has order at most $4 g+2$, cf. [W] Section 2, or Harvey, cf. [Ha] Corollary to Theorem 6 . For each genus $\geq 2$ Wiman also exhibited a surface with such automorphism group: namely $w^{2}=z^{2 g+1}-1$. In a different direction Accola and Maclachlan independently proved the following. Let $n(g)$ denote the maximum of the orders of $\operatorname{Aut}\left(X_{g}\right)$ where $X_{g}$ varies over all compact Riemann surfaces of genus $g$. In $[\mathrm{Ac}],[\mathrm{M}]$ it is proved that $n(g) \geq 8(g+1)$, and for each $g$ there exists a surface admitting an automorphism group of that order, namely $w^{2}=z^{2 g+2}-1$.

In this note we consider the question as to how far are these surfaces determined by the orders of their automorphism groups alone. Our interest is in the "stable" situation, i.e. in investigating the structure of the groups of order $4 g+2$ and $8 g+8$ and the corresponding surfaces for large values of $g$. It turns out that the surfaces of Wiman and Accola-Maclachlan are the "main" members of these families in a certain probabilistic sense. More precisely if $g$ is sufficiently large and is such that $2 g+1$ is not of the form $3 m$ or $9 m$ where all prime divisors of $m$ are congruent to $1 \bmod 3$ then the Wiman surface is the only Riemann surface of genus $g$ which admits an automorphism group of order $4 g+2$. Similarly if $g$ is sufficiently large and is not congruent to $-1 \bmod 4$ then the Accola-Maclachlan surface is the only Riemann surface of genus $g$ which admits an automorphism group of order $8 g+8$. The excluded genera in these statements are genuine exceptions. In the $(4 g+2)$-case (respectively $(8 g+8)$-case) there are precisely 2 (respectively 1 ) additional Riemann surfaces admitting automorphism groups of the respective orders. For more precise statements see Theorems 4.7, 5.5, 6.7.

The above automorphism groups are special cases of the groups of orders $a g+b$ acting on surfaces of genus $g$, with $a+b \neq 0$, where $a, b$ are rational numbers. Whereas in general it would be difficult to obtain the precise statements

Partially supported by an NSF grant, and a PSC-CUNY award. 
as in the above $(4 g+2)$ - and $(8 g+8)$-cases the families of these types would be expected to show some patterns. We hope to investigate this theme in a future paper.

\section{Preliminaries}

2.1. Throughout $X_{g}$ will denote a compact Riemann surface of genus $g$, and $\Sigma_{g}$ the underlying compact orientable surface. While an automorphism group of $X_{g}$ has its usual meaning, by an automorphism group of $\Sigma_{g}$ we simply mean a finite group of orientation-preserving homeomorphisms of $\Sigma_{g}$. A part of the theory of Hurwitz concerning automorphisms of Riemann surfaces is purely topological. We recall it in those terms mainly to set up a notation.

2.2. Let $G$ be a finite group of orientation-preserving homeomorphisms of $\Sigma_{g}$. Then the orbit-space $G \backslash \Sigma_{g}$ is homeomorphic to $\Sigma_{h}$ for some $h$. Let $p: \Sigma_{g} \rightarrow \Sigma_{h}$ be the canonical projection. The fixed points of a non-identity element in $G$ is a discrete (hence finite) set. The stabilizer subgroup of a point is cyclic. Let $S$ be the union of all the fixed points of non-identity elements in $G$. Then $S$ is called the singular set of the $G$-action and $B=p(S)$ the branch set of the $G$-action. The common order of the stabilizer subgroups at the points in $p^{-1}(y), y \in B$, is the branching index $n_{y}$ of $y$. Let $B=\left\{y_{1}, y_{2}, \ldots, y_{r}\right\}$, and $\left\{n_{1}, n_{2}, \ldots, n_{r}\right\}$ be the corresponding indices. The Riemann-Hurwitz relation reads

$$
2-2 g=|G|\left\{2-2 h-\Sigma_{y \in B}\left(1-1 / n_{y}\right)\right\} .
$$

Moreover $G$ has a system of generators $\left\{a_{i}, b_{i}, x_{j}\right\}, 1 \leq i \leq h, 1 \leq j \leq r$, satisfying

$$
\text { order } x_{j}=n_{j} \quad \text { and } \quad \prod_{i=1}^{h}\left(a_{i}, b_{i}\right) \prod_{j=1}^{b} x_{j}=1
$$

and possibly some other relations. The symbol $\left\{h ; n_{1}, n_{2}, \ldots, n_{r}\right\}$ is called the branch data of the $G$-action. When $g \geq 2$ by $(2.2 .1)$ the quantity $\{2-2 h-$ $\left.\Sigma_{y \in B}\left(1-1 / n_{y}\right)\right\}$ is negative. In this case if $G$ is an automorphism group of a Riemann surface $\mathrm{X}_{g}$ then the branch data is the signature of the Fuchsian group which uniformizes this action. When the complex structure is not relevant and the Fuchsian group is considered only as a group of homeomorphisms it will be convenient to call it a topological Fuchsian group.

Conversely given a system of generators $\left\{a_{i}, b_{i}, x_{j}\right\}, 1 \leq i \leq h, 1 \leq j \leq b$, for $G$ satisfying (2.2.2), and $g$ determined by

$$
2-2 g=|G|\left\{2-2 h-\Sigma_{j}\left(1-1 / n_{j}\right)\right\} .
$$

there is a $G$-action on $\Sigma_{g}$ with orbit-space $\Sigma_{h}$ and the branch data of the above form.

2.3. We note the following restrictions for the branch data. 
Proposition. Let $G$ be an automorphism group of $\Sigma_{g}, g \geq 2$. Suppose that $|G|>4(g-1)$. Then the possible branch data are:

i) $(0 ; 2,2,2, n), 3 \leq n$;

ii) $(0 ; 2, m, n), 3 \leq m \leq n$; if $m=3$ then $n \geq 7$, if $m=4$ then $n \geq 5$;

iii) $(0 ; 3, a, n), 3 \leq a \leq 6, a \leq n$; if $a=3$ then $n \geq 4$;

iv) $(0 ; 4,4, n), 3 \leq n$;

v) other finitely many possibilities.

If moreover $|G|>8(g-1)$ then the possible branch data are further reduced to:

vi) $(0 ; 2,3, n), 7 \leq n$.

vii) $(0 ; 2,4, n), 5 \leq n$.

viii) other finitely many possibilities.

These are easy consequences of (2.2.1) and were essentially noted by Hurwitz himself, cf. $[\mathrm{Ac}],[\mathrm{Ha}],[\mathrm{Hu}],[\mathrm{K}],[\mathrm{M}]$. A proof may be left to the reader.

\section{A lemma on finite groups containing a cyclic subgroup of index 3}

3.1. In order to prove our results we need some specific information about finite groups in the title of this section.

Lemma. Let $G$ be a finite group containing a cyclic subgroup $A$ of index 3 . Let $\pi$ be the set of primes $\neq 3$ dividing $|G|$, and $A_{\pi}$ the $\pi$-primary subgroup of A. Then either

I) $G$ is cyclic, or

II) $G$ is noncyclic, $A$ is normal in $G$ and $G$ is either a split extension of $A$ by $Z_{3}$ or a split extension of $A_{\pi}$ by a cyclic 3 -group,

or

III) $G$ is a central extension of the subgroup of index 2 in $A$ by $S_{3}$ where $S_{3}$ denotes the symmetric group on 3 letters.

Proof. Let $\phi$ be the permutation representation of $G$ on $G / A$ and $H$ its kernel. It would be convenient to consider $\phi$ as a homomorphism into $S_{3}$ so that the letter 1 corresponds to the coset [A]. Evidently $H \leq A$. The image of $\phi$ is transitive on three letters so it is either $\langle(1,2,3)\rangle$ or $S_{3}$. In the following we shall denote for a finite group $U$ and a prime $p$ its $p$-Sylow subgroup by $U_{p}$.

Case 1: $\operatorname{im} \phi=\langle(1,2,3)\rangle$. Then $(G: H)=3=(G: A)$. So $H=A$, i.e. $A$ is normal in $G$. Conversely if $A$ is normal in $G$ then it acts trivially on $G / A$, so $A=H$. The conjugation of $A$ by $G$ gives a representation $\sigma$ of $\langle(1,2,3)\rangle$ into Aut $A$. If $G$ is abelian then $\sigma$ is trivial, $G$ is either cyclic or isomorphic to $A \times Z_{3}$ and the lemma follows easily. So we now assume that $G$ is nonabelian and so $\sigma$ is nontrivial. Now $A_{\pi}$ is characteristic in $A$ so it is normal in $G$, and $G$ is a split extension of $A_{\pi}$ by $G_{3}$. If $G_{3}$ is cyclic we are in the case II). Otherwise $G_{3}$ is a 
3 -group containing a cyclic subgroup of index 3 . By the known structure of such groups, cf. [G], Theorem (4.4), $G_{3}$ is then a split extension of a cyclic subgroup by $Z_{3}$. But then $G$ contains an element of order 3 not lying in $A$. So $G$ is a split extension of $A$ by $Z_{3}$.

Case 2: $\operatorname{im} \phi=S_{3}$. Now $H$ is a subgroup of index 2 in $A$. The conjugation of $H$ by $G$ gives a representation $\sigma$ of $S_{3}$ into Aut $H$. Since Aut $H$ is abelian the subgroup $\langle(1,2,3)\rangle$ must act trivially on $H$. Let $K=\phi^{-1}(\langle 1,2,3\rangle)$. Then $K$ centralizes $H$. Now $A=\phi^{-1}(\langle(2,3)\rangle)$ also centralizes $H$, and $G=\langle K, A\rangle$. So $G$ centralizes $H$. So we are in case III). $\square$

\section{Automorphism groups of order $4 g+2$}

4.1. Let $G$ be an automorphism group of $\Sigma_{g}, g \geq 2$ of order $4 g+2$. So the possible branch data are either of the type i) to iv) listed in 2.3 or other finitely many restrictions which are not listed there. Since we are willing to consider only the large values of $g$ these finitely many possibilities have no effect since each of them determines at most one value of $g$ as can be seen easily from (2.2.1). Our first aim now is to determine the structure of these groups.

4.2. We first prove that the cases $(0 ; 3,3, n),(0 ; 3,4, n),(0 ; 3,5, n)$ and $(0 ; 4,4, n)$ cannot occur. Indeed $(0 ; 3,4, n)$ and $(0 ; 4,4, n)$ do not occur simply because 4 is not a divisor of $4 g+2$. As for $(0 ; 3,5, n)$ let us write $(2.2 .1)$ in the form

$$
\frac{2 g-2}{4 g+2}=1-\frac{1}{3}-\frac{1}{5}-\frac{1}{n} \text {. }
$$

As observed above, each $n$ uniquely determines $g$. Hence as $g$ tends to infinity so does $n$. But the left hand side tends to $\frac{1}{2}$ whereas the right hand side tends to $7 / 15$. So $(0 ; 3,5, n)$ cannot occur. A similar argument shows that the case $(0 ; 3,3, n)$ also cannot occur.

4.3. Now consider the case ii) of the branch data listed in (2.3). The equation (2.2.1) in this case reduces to

$$
3=\frac{4 g+2}{m}+\frac{4 g+2}{n} .
$$

Since $m, n$ are divisors of $4 g+2$, say $m u=n v=4 g+2$, we get $u+v=3$. At least one of $u, v$ must be 1 . But this means that $G$ must be cyclic. Now in the remaining two cases $(0 ; 3,6, n)$ and $(0 ; 2,2,2, n)$ from $(2.2 .1)$ we get $n=(4 g+2) / 3$. This means that $G$ contains a cyclic subgroup of index 3 .

4.4. We now apply the lemma in Section 3 and note further restrictions on $G$. We suppose that $G$ is not cyclic. We first prove that the case III) of (3.1) cannot occur. Suppose it did. Then $G$ is a central extension of a cyclic subgroup $H$ by $S_{3}$. Here the order of $H$ is $(2 g+1) / 3$. 
Assertion 1. $G$ is isomorphic to a direct product of $H$ and $S_{3}$.

Proof. Let $\phi$ be the homomorphism of $G$ onto $S_{3}$ which defines the extension, and $K=\phi^{-1}(\langle(1,2,3)\rangle)$. Then $K$ has order $2 g+1$ and since $H$ is central we see that $K$ is in fact either cyclic or isomorphic to $H+Z_{3}$. Also $G=K \rtimes Z_{2}$, where $Z_{2}$ must act nontrivially on $K$ since otherwise $G$ would be abelian. But since $H$ is central and has index 3 in $K$ it contains the $p$-primary parts of $K$, where $p$ is a prime $\neq 3$. So the $Z_{2}$-action must be nontrivial on the 3 -primary part of $K$ and otherwise trivial on the other $p$-primary parts, $p \neq 3$. If $K$ is isomorphic to $H+Z_{3}$ then clearly $G$ is isomorphic to a direct product of $H$ and $S_{3}$ as claimed. Otherwise $K$ is cyclic and $K_{3}$, i.e. the 3-Sylow subgroup of $K$, must be cyclic of order at least 9 , and $H_{3}$ is a cyclic subgroup of index 3 in $K_{3}$. But there is no nontrivial $Z_{2}$-action on a cyclic 3 -group which is trivial on a subgroup of index 3 . This contradiction shows that $K$ is isomorphic to $H+Z_{3}$ and $G$ is isomorphic to a direct product of $H$ and $S_{3}$. $\square$

So $H$ is also a homomorphic image of $G$. In either of the cases of $(0 ; 3,6, n)$ or $(0 ; 2,2,2, n)$-branch data $G$ is generated by at most 3 elements of order at most 6 . So the order of $H$, and hence also the order of $G$, is bounded. So such actions do not exist for large genera.

4.5. It remains to consider the case II) of Lemma 3.1, i.e. $G$ is a semidirect product of a cyclic subgroup $A$ by $Z_{3}$, where $Z_{3}$ acts nontrivially on $A$, or $G$ is a split extension of $A_{\pi}$ by a cyclic 3 -group. We note at once that the case of the branch data $(0 ; 2,2,2, n)$ cannot occur, for $Z_{3}$ which is a homomorphic image of $G$ is not generated by elements of order 2 . So the branch data must be $(0 ; 3,6, n)$.

Assertion 2. Let $p$ be a prime divisor of $|G|, p \neq 2,3$. Then $p \equiv 1 \bmod 3$. Also $G_{3}$ is isomorphic to $Z_{3}$ or $Z_{3} \times Z_{3}$.

Proof. Let $p$ be a prime divisor of $|G|, p \neq 2,3$. Then $A_{p}=G_{p}$. Since $A_{p}$ is a characteristic subgroup of $A$ we see that $A_{p}$ is normal in $G$, and $G$ is a semidirect product of $A_{p}$ by a subgroup $L$ of order not divisible by $p$. Now suppose if possible that $p \equiv-1 \bmod 3$. Then $A_{p}$ does not admit a nontrivial action by $Z_{3}$. Since $A$ commutes with $A_{p}$ it is clear that $G$ is in fact a direct product of $A_{p}$ and $L$. So $A_{p}$ is also a homomorphic image of $G$. But it cannot be generated by elements of order 3 and 6 . This contradiction shows that every prime divisor of $|G|$ different from 2 and 3 is $\equiv 1 \bmod 3$.

Now let $\pi$ be the set of prime divisors of $|G|$ different from 3 . Then by an analogous argument the $\pi$-primary part $A_{\pi}$ of $A$ is normal in $G$ and $G$ is isomorphic to a semidirect product of $A_{\pi}$ by $G_{3}$. So $G_{3}$ is a 3-group with a cyclic subgroup of index 3 and being a homomorphic image of $G$ it is generated by at most two elements of order 3 . From the wellknown structure of 3 -groups with a cyclic subgroup of index 3 it follows that $G_{3}$ is isomorphic to $Z_{3}$ or $Z_{3} \times Z_{3}$. .

4.6. The above argument also shows that for each prime divisor $p \equiv 1 \bmod 3$ of $|G|$ we must have $Z_{3}$ acting nontrivially on $A_{p}$. This then determines the 
isomorphism type of $G$ as noted down in part II) of the theorem below. Notice that $|G|$ is $6 m$ or $18 m$ where every prime divisor of $m$ is congruent to $1 \bmod 3$.

4.7. Theorem I). For $g$ sufficiently large the Riemann surfaces of genus $g$ admitting an automorphism group of order $4 g+2$ are classified as follows. There is a unique Riemann surface admitting a cyclic automorphism group of order $4 g+2$. If $2 g+1=3 m$ or $9 m$ where every prime divisor of $m$ is congruent to $1 \bmod 3$ then there are 2 Riemann surfaces admitting a noncyclic automorphism group of order $4 g+2$.

II) The isomorphism type of a noncyclic automorphism group $G$ is uniquely determined as follows: If $2 g+1=3 m$, respectively $9 m$, where every prime divisor of $m$ is congruent to $1 \bmod 3$ then $G \approx Z_{a} \times\left(B \rtimes Z_{3}\right)$ where $a=2$ (respectively 6 ), $B$ is a cyclic subgroup of order $m$, and for each prime factor $p$ of $|B|, Z_{3}$ acts nontrivially on $B_{p}$.

(Note: i) Two groups $G_{1}$, respectively $G_{2}$ of homeomorphisms of topological spaces $X_{1}$, respectively $X_{2}$, are considered equivalent if there is a homeomorphism $h$ from $X_{1}$ to $X_{2}$ such that $h G_{1} h^{-1}=G_{2}$. In the context of Riemann surfaces one would require $h$ to be a biholomorphic map.

ii) More succinctly $G$ may be described by its presentation as follows. Write $n=(4 g+2) / 3$. Then

$$
G=\left\langle z_{0}, y_{0} \mid z_{0}^{n}=y_{0}^{3}=e, y_{0} z_{0} y_{0}^{-1}=z_{0}^{r}\right\rangle
$$

where

a) if $|G|=6 m$ then $r^{3} \equiv 1(\bmod n),(r-1, n)=2$, or

b) if $|G|=18 m$ then $r^{3} \equiv 1(\bmod n),(r-1, n)=6$.)

Proof. The assertion II) is already proved above. If $G$ is cyclic the assertion I) was proved in $[\mathrm{K}]$, in fact for all $g \geq 2$. (The following argument could also be used to offer a still different proof of the uniqueness statement there.) We now assume that $G$ is not cyclic.

We first note that the assertion I) is equivalent to the underlying purely topological assertion: there are two inequivalent $G$-actions on $\Sigma_{g}$. Indeed equipping a structure of a Riemann surface on $\Sigma_{g} / G$ and "pulling" it back to $\Sigma_{g}$ we see that $G$ can be made into a group of holomorphic automorphisms of some complex structure on $\Sigma_{g}$. Since the action has 3 branch points and the complex structure on a thrice-punctured sphere is unique we see that the $G$-action uniquely determines the complex structure on $\Sigma_{g}$. So if there are two distinct (i.e. nonbiholomorphic) Riemann surfaces admitting $G$-actions, the actions must be topologically inequivalent. Conversely if the same Riemann surface admits two distinct $G$-actions then its automorphism group would have order larger than $4 g+2$. This would give a proper inclusion of the triangle group with signature $(0 ; 3,6,(4 g+2) / 3)$ into another Fuchsian group. However no such proper inclusion exists, thanks to the complete classification of such inclusions by Singerman, cf. [S], Theorems 1 and 2. 
Now a $G$-action on $\Sigma_{g}$ with a given branch data $\{0 ; 3,6, n\}$ gives an inclusion of $\pi=\pi_{1}\left(\Sigma_{g}\right)$ into the triangle group $\Delta=\Delta_{\{0 ; 3,6, n\}}$ as a normal subgroup so that $\Delta / \pi \approx G$. We take

$$
\Delta=\left\{\xi, \eta, \zeta \mid \xi^{3}=\eta^{6}=\zeta^{n}=\xi \eta \zeta=e\right\}
$$

Since $\Delta$ is a maximal Fuchsian group, cf. [S], we see that the $G$-actions on $\Sigma_{g}$ are classified by normal torsionfree subgroups $\approx \pi$. Such normal subgroups arise precisely as kernels of homomorphisms of $\Delta$ onto $G$ which map $\xi, \eta, \zeta$ onto elements $x, y, z$ of orders $3,6, n$ respectively so that $x y z=e$. Let us call a triple $(x, y, z)$ of elements in $G$ of orders $3,6, n$ respectively a Hurwitz system if $x, y, z$ generate $G$ and $x y z=e$. Obviously any Hurwitz system determines canonically a homomorphism of $\Delta$ into $G$, the kernel $\approx \pi$ and a $G$-action on $\Sigma_{g}$. Two Hurwitz systems which differ by an automorphism of $G$ obviously determine the same $G$-actions. The map

\section{\{Hurwitz systems $\} /$ Aut $G \mapsto\left\{G-\right.$ actions on $\left.\Sigma_{g}\right\}$}

which can be defined for arbitrary automorphism groups of compact orientable surfaces is in general surjective but not necessarily injective. However in our special case we shall now show it to be injective and that there are precisely 2 elements in either set.

To this end we take $G$ as in (4.7.1). It is clear that $A=\left\langle z_{0}\right\rangle$ is a normal subgroup of $G$ of index 3 and it coincides with the the group generated by the center of $G$ and the commutator subgroup of $G$. The commutator subgroup coincides with $\left\langle z_{0}^{r-1}\right\rangle$. The elements of $G$ outside $A$ are of order 3 or 6 . Any Hurwitz system arising from a $G$-action on $\Sigma_{g}$ has the form $(x, y, z)$ where $z=z_{0}^{s}$, $(s, n)=1, y$ is an element of order 3 outside $A$ and $x=(y z)^{-1}$. One sees that the possible forms of $y$ are $y_{0}^{j} z_{0}^{t}, j=1$ or 2 and $t$ even. Now note that both $\left\{z_{0} \mapsto z_{0}^{s}\right.$, $\left.y_{0} \mapsto y_{0},(s, n)=1\right\}$, and $\left\{z_{0} \mapsto z_{0}, y_{0} \mapsto y_{0} z_{0}^{t}, t\right.$ even $\}$ define automorphisms of $G$. So modulo Aut $G$ every Hurwitz system is equivalent to $\left(\left(y_{0} z_{0}\right)^{-1}, y_{0}, z_{0}\right)$ or $\left(\left(y_{0}^{2} z_{0}\right)^{-1}, y_{0}^{2}, z_{0}\right)$. One easily checks that these systems are indeed inequivalent. Moreover we claim that they define two distinct normal subgroups of $\Delta$. For if the same normal subgroup, say $\Phi$, is defined by both the Hurwitz systems then $\eta \zeta \eta^{-1} \zeta^{r}$ and $\eta \zeta \eta^{-1} \zeta^{r^{2}}$ would lie in $\Phi$. But then $\zeta^{r^{2}-r}$ would lie in $\Phi$, and the order of the image of $\zeta$ in $G$ would be smaller than $n$. So the two Hurwitz systems define two distinct normal subgroups of $\Delta$. As observed earlier this means that the $G$-actions are distinct and so there are two distinct Riemann surfaces of genus $g$ admitting actions by a noncyclic subgroup of order $4 g+2$. 


\section{Accola-Maclachlan surfaces}

5.1. By looking at the list in 2.3 and the argument in 4.2 it follows that the only possible branch data is $(0 ; 2,4,2 g+2)$. Write $n$ for $2 g+2$. We first need to consider the groups $G$ of order $4 n$ generated by two elements $x, y$ of orders $n$ and 4 respectively so that $x y$ has order 2 . So

$$
(x y)^{2}=e, \quad \text { or } \quad x y=y^{-1} x^{-1}, \quad \text { or } \quad y x y^{-1}=x^{-1} y^{2} .
$$

Write $A=\langle x\rangle$.

5.2. Assertion. $A$ is not normal in $G$, and $\langle y\rangle$ is a complement of $A$.

Proof. If $A$ is normal then the last relation in (5.1.1) shows that $y^{2}$ would lie in $A$. But then $x, y$ would generate a subgroup of order at most $4 g+4$. So $A$ is not normal in $G$. Also $\langle y\rangle$ does not intersect $A$ nontrivially for otherwise $y$ being only of order $4, y^{2}$ would have to lie in $A$ leading to the same contradiction. So $\langle y\rangle$ is a complement of $A$. 口

5.3. Consider the permutation representation $\phi$ of $G$ on $G / A$. We regard $\phi$ as a homomorphism of $G$ into $S_{4}$ where the latter is a symmetric group on $\{1,2,3,4\}$ and the coset $[A]$ is identified with the symbol 1 . Notice that $\phi(y)$ must be a 4-cycle for otherwise it would be a 2-cycle or a product of two 2cycles and $y^{2}$ would be in $\langle x\rangle$. By renumbering if necessary we may take $\phi(y)=$ $(1,2,3,4)$. Now we claim that $\phi(x)$ must be a 2 -cycle. For if possible assume the contrary. Since $\phi(x)$ fixes 1 and does act nontrivially (since $\langle x\rangle$ is not normal in $G$ ), it would follow that $\phi(x)$ must be a 3 -cycle. Then the group generated by $\phi(x)$ and $\phi(y)$ would have order divisible by 12 , and since the alternating group does not contain a 4 -cycle this group has to be $S_{4}$. This is not possible for the stabilizer subgroup of $S_{4}$ at 1 is not cyclic and would not be contained in $\phi(A)$. So $\phi(x)$ must be a 2-cycle. Also by the second relation in (5.1.1) we have $\phi(x) \phi(y)(1)=\phi(x)(2)=\phi\left(y^{-1}\right) \phi\left(x^{-1}\right)(1)=4$. So $\phi(x)=(2,4)$. It follows that $\phi\left(y^{2}\right)$ commutes with $\phi(x)$ and so $y^{2}$ normalizes $A$.

It is clear that $\operatorname{im} \phi$ is isomorphic to $D_{8}$, i.e. the dihedral group with 8 elements. So the kernel of $\phi$ must have index 2 in $A$. In other words $K=$ $\operatorname{ker} \phi=\left\langle x^{2}\right\rangle$. Now the conjugation action of $G$ on $K$ factors through the action of $D_{8}$. Since $K$ is cyclic Aut $K$ is abelian. Now the commutator quotient of $D_{8}$ is the Klein 4-group, i.e. isomorphic to $Z_{2} \times Z_{2}$. In particular the conjugation of $K$ by $y$ is of order 2 . So $y^{2}$ acts trivially on $K$. In other words, $y^{2}$ centralizes $\left\langle x^{2}\right\rangle$. of $G$.

5.4. Assertion. If $g \equiv 0,1$, or $2 \bmod 4$, then $y^{2}$ actually lies in the center

Proof. Suppose first that $g$ is even. Then $A \approx Z_{2} \times K$ and $K$ has odd order. Since the conjugation action of $y^{2}$ on $K$ is trivial it is clear that it is actually trivial on $A$ as well. So $y^{2}$ lies in the center of $G$. Next suppose that $g \equiv 1$ 
$\bmod 4$. Then $A \approx Z_{4} \times Z_{(g+1) / 2}$ and the odd order factor is contained in $K$. If the action of $y^{2}$ on $A$ were not trivial it would have to be nontrivial on the $Z_{4}$-factor. We can write the generator $x^{-1}$ of $A$ in the form $x_{1} x_{2}$ where $x_{1}$ has order 4 and $x_{2}$ has odd order. But then $x^{-1} y^{2}=x_{1} x_{2} y^{2}=x_{2} y^{2} x_{1}^{-1}$ and it is easy to see that the order of $x^{-1} y^{2}$ would be $g+1$. But by the third relation in (5.1.1) $x^{-1} y^{2}$ is conjugate to $x$ and has order $2 g+2$. This contradiction shows that the action of $y^{2}$ on $A$ must be trivial. So $y^{2}$ lies in the center of $G$. व

5.5. Theorem. Let $X_{g}$ be a Riemann surface admitting an automorphism group $G$ of order $8 g+8$, where $g$ is sufficiently large. If $g \equiv 0,1$, or $2 \bmod 4$, (or more generally in the notation of (5.4) if $y^{2}$ lies in the center of $G$ ) then $X_{g}$ is uniquely determined. It is hyperelliptic and is given by $w^{2}=z^{2 g+2}-1$. The group $G$ is its full automorphism group. As an abstract group this group is isomorphic to

$$
\left(Z_{2 g+2}+Z_{2}\right) \rtimes Z_{4} / B
$$

Here the action of $Z_{4}=\langle y\rangle$ on $Z_{2 g+2}+Z_{2}=\langle x\rangle+\langle t\rangle$ is given by $y x y^{-1}=x^{-1} t$, $y t y^{-1}=t$, and $B=\left\langle y^{2} t\right\rangle$.

Proof. We know that $X_{g} / G$ is a sphere with three branch points-say, $a, b$, $c$-of index 2, 4, and $2 g+2$ respectively. Let $p: X_{g} \rightarrow X_{g} / G$ be the canonical projection. We may take $\langle y\rangle$ to be the stabilizer of a point in $p^{-1}(b)$. Now $p^{-1}(b)$ has $2 g+2$ points. Also $A$ is a complement of $\langle y\rangle$ so it does not intersect any conjugate of $\langle y\rangle$. Since $A$ has $2 g+2$ elements it follows that $A$ acts simply transitively on $p^{-1}(b)$. Let $\psi$ be this permutation representation. By numbering the points in $p^{-1}(b)$ by $\{1,2, \ldots, 2 g+2\}$ suitably we may take $\psi(y)(1)=1$, and $\psi(x)=(1,2, \ldots, 2 g+2)$. Since under our hypothesis $y^{2}$ commutes with $x$ and $\psi\left(y^{2}\right)(1)=1$, we see that $\psi\left(y^{2}\right)=e$. In other words $y^{2}$ has $2 g+2$ fixed points. It is wellknown that this is the maximum number of fixed points for a nontrivial automorphism of a surface, and when such an automorphism exists the corresponding Riemann surface is hyperelliptic, and the automorphism is the hyperelliptic involution. It is also wellknown that the hyperelliptic involution is central in the full automorphism group of the surface.

From (5.1.1) and the above results the isomorphism type of $G$ is clearly as described above. The hyperelliptic involution is $y^{2}$. Also $y^{2}=t$. Let $G /\langle t\rangle=H$ which is easily seen to be isomorphic to the dihedral group $D_{4 g+4}$. Let $C$ be the normal cyclic subgroup of order $2 g+2$ in $H$. It acts on the Riemann sphere. Since up to conjugacy in the full Möbius group a cyclic subgroup is determined uniquely by its order we may choose the coordinate $z$ so that the fixed points of $C$ are at 0 and $\infty$ and the fixed points of elements of order 2 in the nonidentity coset of $C$ in $H$, which form a single $C$-orbit, are at the $2 g+2$-th roots of unity. Our original Riemann surface is obtained as the 2 -sheeted cover which is branched at 
these roots of unity. This implies the equation for the surface as given above in a wellknown manner.

Finally the full automorphism group of this surface is also $G$. For otherwise we shall get a proper inclusion of the triangle group with signature $\{0 ; 2,4,2 g+2\}$ into another Fuchsian group. No such inclusion exists according to Singerman, cf. [S], Theorems 1 and 2. 口

\section{The exceptional automorphism groups of order $8 g+8$}

6.1. In view of 5.5 it remains to investigate the case when $g \equiv-1 \bmod 4$. In this case indeed there are other possible automorphism groups of order $8 g+8$ and correspondingly other Riemann surfaces. We continue with the analysis of the group in the notation used in Section 5. Write $2 g+2=2^{k} m$ where $m$ is odd and $k \geq 3$.

6.2. Write the generator $x^{-1}$ of $A$ in the form $x_{1} x_{2}$ where $x_{1}$ has order $2^{k}$ and $x_{2}$ has order $m$. The conjugation action of $y^{2}$ on $\left\langle x_{2}\right\rangle$ is trivial since $\left\langle x_{2}\right\rangle$ is contained in $\left\langle x^{2}\right\rangle$. As is wellknown there are four possible $Z_{2}$-actions on $\left\langle x_{1}\right\rangle$; namely i) $x_{1} \mapsto x_{1}$, ii) $x_{1} \mapsto x_{1}^{-1}$, iii) $x_{1} \mapsto x_{1}^{2^{k-1}-1}$, and iv) $x_{1} \mapsto x_{1}^{2^{k-1}+1}$. Now we observed in 5.4 that $x^{-1} y^{2}$ is conjugate to $x$ and so has order $2 g+2$. This condition shows that $x_{1} y^{2}$ has order $2^{k}$. It is easily checked that only the actions i) and iv) are consistent with this requirement. In the case i) $y^{2}$ is central and this is taken care of in 5.5. So now we assume that the case iv) occurs. In this case the 2-group $\left\langle x_{1}, y^{2}\right\rangle$ is isomorphic to a semidirect product of a cyclic group by $Z_{2}$ known as a twisted semidihedral group usually denoted by $S^{*} D_{2^{k+1}}$. Our aim now is to determine the possible isomorphism types of $G$ assuming that it exists. Fortunately it turns out that there is only one isomorphism type.

6.3. First let $L=\left\langle x, y^{2}\right\rangle$. Clearly $L$ contains $A$ as a normal subgroup of index 2 , and $G$ contains $L$ as a normal subgroup of index 2 . Let $\pi$ be the set of all odd prime divisors of $|G|$. Then $A_{\pi}$, i.e. the $\pi$-primary part of $A$, is contained in $\left\langle x^{2}\right\rangle$ and so it is central in $L$. So $L$ is an internal direct product of $A_{\pi}$ by $L_{2}$, where the latter is isomorphic to $S^{*} D_{2^{k+1}}$. This determines the isomorphism type of $L$. It is convenient to write down its following presentation. Notice that $g+2$ is the unique natural number less than $2 g+2=2^{k} m$ which is $\equiv 2^{k-1}+1$ $\bmod 2^{k}$ and $\equiv 1 \bmod m$. Then writing $u=y^{2}$,

$$
L=\left\langle x, u \mid x^{2 g+2}=u^{2}=e, u x u=x^{g+2}\right\rangle .
$$

6.4. Next notice that the element $y x$ of $G$ is not in $L$ and has order 2. So $G$ is an internal semidirect product of $L$ by $\langle y x\rangle$. The conjugation action of $y x$ on $L$ would be determined by

$$
x \mapsto y x y^{-1}=x^{-1} y^{2}=x^{-1} u, \quad u \mapsto y x u y x=y x y^{-1} x=x^{-1} u x=x^{g+1} u .
$$


Now the point is that the order of $x^{\prime}=x^{-1} u$, respectively $u^{\prime}=x^{g+1} u$, is indeed $2 g+2$, respectively 2 , and $u^{\prime} x^{\prime} u^{\prime}=x^{g+2}$, and moreover

$$
x \mapsto x^{-1} u, \quad u \mapsto x^{g+1} u,
$$

is indeed an automorphism of $L$ of order 2 as can be checked from its presentation (6.3.1). So such $G$ indeed exists. Its presentation may be given by

$$
G=\left\langle x, y \mid x^{2 g+2}=y^{4}=(y x)^{2}=e, y^{2} x y^{2}=x^{g+2}\right\rangle .
$$

By the Hurwitz procedure, cf. 2.2 , it is clear that $G$ acts on $\Sigma_{g}$ with branching data $(0 ; 2,4,2 g+2)$.

6.5. For determining all actions of $G$ it is important to know the orders of the elements in $G$. Since $\langle y\rangle$ is a complement of $\langle x\rangle$ every element of $G$ can be written as $y^{i} x^{j}$ where $0 \leq i \leq 3,0 \leq j \leq 2 g+1$. Note the following properties:

a) $y^{2}$ commutes with $x^{2}$.

b) $y x y^{-1}=x^{-1} y^{2}=y^{2} x^{-g-2}=y^{2} x^{g}, y^{-1} x y=y^{2} x^{-1}=x^{g} y^{2}$.

c) $y^{2} x^{j} y^{-2}=x^{j(g+2)}$.

d) $y^{-1} x^{2} y=y x^{2} y^{-1}=x^{-1} y^{2} x^{-1} y^{2}=x^{-1} x^{g}=x^{g-1}$; hence $y^{-1} x^{2 j} y=y x^{2 j} y^{-1}=x^{j(g-1)}$.

\subsection{Lemma.}

i) The orders of both $y x^{2 j}$ and $y^{-1} x^{2 j}$ are 4 .

ii) The order of $y x^{2 j+1}$ is 2 or 4 according as $j$ is even or odd.

iii) The order of $y^{-1} x^{2 j+1}$ is 2 or 4 according as $j$ is odd or even.

iv) The orders of both $y^{2} x^{r}$ and $x^{r}$ are same.

Proof. i): Notice that $y x^{2 j} y x^{2 j}=x^{j(g-1)} y^{2} x^{2 j}=x^{j(g+1)} y^{2}$. Now $x^{j(g+1)}$ has order 1 or 2 according as $j$ is even or odd. In either case $\left(y x^{2 j}\right)^{2}$ has the same order as $y^{2}$. Hence the order of $y x^{2 j}$ is 4 . The proof for $y^{-1} x^{2 j}$ is similar. ii), iii): Next

$$
\begin{aligned}
y x^{2 j+1} y x^{2 j+1} & =y x^{2 j} y^{-1} y x y^{-1} y^{2} x^{2 j+1} \\
& =x^{j(g-1)} x^{-1} y^{2} y^{2} x^{2 j+1}=x^{j(g-1)-1+2 j+1}=x^{j(g+1)} .
\end{aligned}
$$

So the order of $y x^{2 j+1}$ is 2 or 4 according as $j$ is even or odd. On the other hand

$$
\begin{aligned}
y^{-1} x^{2 j+1} y^{-1} x^{2 j+1} & =y^{-1} x^{2 j} y y^{-1} x y y^{2} x^{2 j+1} \\
& =x^{j(g-1)} y^{2} x^{-1} y^{2} x^{2 j+1}=x^{j(g-1)+g+2 j+1}=x^{(j+1)(g+1)} .
\end{aligned}
$$

So the order of $y^{-1} x^{2 j+1}$ is 2 or 4 according as $j$ is odd or even. iv): Now

$$
y^{2} x^{r} y^{2} x^{r}=x^{r(g+3)}=x^{r(g+1)} x^{2 r}
$$

which has the same order as $x^{2 r}$. So the order of $y^{2} x^{r}$ is equal to that of $x^{r}$. 
6.7. The possible Hurwitz systems are $(\xi, \eta, \zeta)$ where $\xi, \eta, \zeta$ have orders $2 g+2,4,2$ respectively so that $\xi, \eta, \zeta$ generate $G$ and their product $\xi \eta \zeta=e$. From the above lemma we see that the possibilities for $(\xi, \eta)$ are of two types:

A) $\left(x^{r}, y x^{s}\right)$ or $\left(y^{2} x^{r}, y^{-1} x^{s}\right)$ where $(r, 2 g+2)=1, r+s \equiv 1 \bmod 4$.

B) $\left(x^{r}, y^{-1} x^{s}\right)$ or $\left(y^{2} x^{r}, y x^{s}\right)$ where $(r, 2 g+2)=1, r+s \equiv-1 \bmod 4$.

From the presentation (6.4.1) it is not difficult to check that there are automorphisms of $G$ carrying $(x, y)$ to any of the above Hurwitz systems. So from the discussion in 4.7 it follows that the $G$-action is unique upto topological equivalence. Since the quotient is a sphere with three branch points it follows again that the corresponding Riemann surface admitting this action is unique. Finally again appealing to $[\mathrm{S}]$ it follows that this Riemann surface is distinct from the one in Theorem 5.5. Also for the same reason $G$ is the full automorphism group of the surface. To summarize:

Theorem. For large values of $g$ if $g \equiv 0,1,2 \bmod 4$ then there is a unique Riemann surface (given by $w^{2}=z^{2 g+2}-1$ ) admitting an automorphism group of order $8 g+8$. If $g \equiv-1 \bmod 4$ then in addition to the above one there is precisely one more such Riemann surface. In both cases the full automorphism group coincides with the automorphism group of order $8 g+8$. Their isomorphism types are given by their presentations (5.5.1) and (6.4.1).

\section{References}

[Ac] Accola, R.D.M.: On the number of automorphisms of a closed Riemann surface. - Trans. Amer. Math. Soc. 131, 1968, 398-408.

[G] Gorenstein, D.: Finite groups. - Harper and Row, New York, 1968.

[Ha] HarveY, W.J.: Cyclic groups of automorphisms of a compact Riemann surface. - Quarterly J. Math. 17, 1966, 86-97.

[Hu] Hurwitz, A.: Über algebraische Gebilde mit eindeutigen Transformationen in sich. Math. Ann. 41, 1893, 403-442.

[M] Maclachlan, C.: A bound for the number of automorphisms of a compact Riemann surface. - J. London Math. Soc. 44, 1969, 265-272.

[K] KUlKarNi, R.S.: Riemann surfaces admitting large automorphism groups. - Preprint.

[S] Singerman, D.: Finitely maximal Fuchsian groups. - J. London Math. Soc. (2) 6, 1972, 29-38.

[W] Wiman, A.: Über die hyperelliptischen Curven und diejenigen von Geschlechte $p=3$ welche eindeutige Transformationen in sich zulassen. - Bihang till K. Svenska Vet.Akad. Handlingar, Stockholm 1895-6, bd. 21, 1-23.

City University of New York

Graduate Center

Department of Mathematics

33, W. 42nd Street

New York, N.Y. 10036

U.S.A.

Received 15 December 1989 